Service social

\title{
Solidarités invisibles et prise en charge de la communauté par elle-même
}

\section{Andrée Fortin}

Volume 41, numéro 1, 1992

L'avenir des services ou services d'avenir

URI : https://id.erudit.org/iderudit/706555ar

DOI : https://doi.org/10.7202/706555ar

Aller au sommaire du numéro

Éditeur(s)

École de service social de l'Université Laval

ISSN

1708-1734 (numérique)

Découvrir la revue

Citer cet article

Fortin, A. (1992). Solidarités invisibles et prise en charge de la communauté par elle-même. Service social, 41(1), 7-27. https://doi.org/10.7202/706555ar
Résumé de l'article

Le nombre considérable d'associations au Québec, et leur augmentation depuis une vingtaine d'années, pourrait laisser croire à une effervescence communautaire. Par ailleurs, l'époque actuelle est souvent caractérisée par l'individualisme. Sans nier l'existence d'un narcissisme individuel ou collectif, l'auteure trace les contours d'une mobilisation communautaire à la faveur de cette vie associative et met en évidence la dialectique entre la fermeture des associations sur elles-mêmes et leur ouverture sur la communauté, la mise en oeuvre de solidarités communautaires à la faveur de la sociabilité privée, prise en charge de la communauté par elle-même et qui passe trop souvent inaperçue. 


\section{ARTICLES}

Andrée Fortin, professeure, Département de sociologie, Université Laval.

\section{Solidarités invisibles et prise en charge de la communauté par elle-même ${ }^{1}$}

Andrée Fortin

Le nombre d'associations actives au Québec, plus de 24500 en 1989, et leur augmentation depuis une vingtaine d'années, pourrait laisser croire à une effervescence communautaire. Par ailleurs, l'époque actuelle est souvent caractérisée par le narcissisme, l'individualisme ou le "cocooning ". Illusion d'optique ? Si oui, de quel côté : de celui qui met l'accent sur la vigueur associative ou de celui du narcissisme? Ces deux visions se réconcilient-elles dans celle d'un narcissisme collectif à l'intérieur des associations? Nous posons plutôt l'hypothèse d'une mobilisation communautaire s'appuyant à la fois sur l'association comme groupe et sur les individus qui en sont membres. Nous tenterons ici de mettre en évidence la dialectique entre la fermeture des associations sur. elles-mêmes et leur ouverture sur la communauté ainsi que la mise en œuvre de solidarités communautaires à la faveur de la sociabilité privée, prise en charge de la communauté par elle-même qui passe souvent inaperçue.

Avant toute chose, des précisions s'imposent sur la vie associative. Langlois (Langlois et al., 1990 : 108-109) fait état de la création de 32521 associations entre 1973 et 1989. Toutes n'ont pas survécu, mais si en 1973 il y avait 6103 associations actives au Québec, en 1989 elles étaient 24 512. Pour une plus juste idée de 
I'effervescence communautaire, il faut ajouter à ce total les associations bona fide, non recensées dans ces données, par exemple certaines ligues sportives (quilles, dards, etc.), ainsi que les quelque 1200 coopératives d'habitation qui, à plusieurs égards, par leur fonctionnement et leurs objectifs, se rattachent au secteur associatif.

Plus révélatrice que le nombre d'associations est leur répartition. Langlois (Langlois et al., 1990 : 105-106) en propose une typologie à onze catégories : 1- sportives, 2- religieuses, 3- politiques (organisations et partis; groupes populaires), 4- liens sociaux et communautaires (associations amicales; clubs sociaux; clubs de l'âge d'or; anciens élèves), 5- loisirs sociaux et culturels (clubs communautaires; radio amateur; YMCA et YWCA; scouts; spectacles), 6- action sociale (santé et services sociaux; fondations privées), 7 - linguistiques et nationales (ethniques, Société Saint-JeanBaptiste), 8- parents et étudiants, 9- propriétaires et locataires, 10- gens d'affaire, 11- promotion d'intérêts (professionnels, commerciaux; syndicats). Parmi les associations actives en 1989, 29,1\% concernent les liens sociaux et communautaires, 21,6 \% s'occupent de loisirs sociaux et culturels. C'est donc près des trois quarts des associations œuvrant actuellement dans le domaine de la vie sociale et communautaire au sens large qui misent sur le plaisir d'être ensemble 2 . Par opposition, les autres associations poursuivent un but en dehors d'elles-mêmes et ce but, plutôt que le plaisir d'être ensemble, est ce qui préside à leur formation. Ainsi les associations à caractère politique, comprenant les groupes populaires, de 1,9\% des associations actives en 1973, sont passées à $2,5 \%$ en 1989. Les groupes d'entraide dont il est beaucoup question depuis quelques années (Brault et Saint-Jean, 1990; Godbout, 1990) sont difficiles à repérer dans la typologie proposée par Langlois; elles correspondent grosso modo à celles regroupées sous l'étiquette " action sociale » représentant $7,3 \%$ du total en 1973 et $9,7 \%$ en 1989. Ces augmentations, proportionnellement faibles, ne doivent pas faire oublier que le nombre absolu de groupes a augmenté.

En résumé, les groupes à caractère politique, peu nombreux proportionnellement, le sont plus que dans les années 70 , alors qu'on en faisait grand cas; en matière de visibilité sociale, ces années-ci, ce sont les groupes d'entraide qui attirent toute l'attention. II n'en demeure pas moins que les associations de "loisir", par opposition aux associations politiques ou d'entraide, sont beaucoup plus nombreuses'. Ne s'occupent-elles que de loisir ou mettent-elles en branle des solidarités? 
S'il n'est pas possible de conclure d'emblée à la mobilisation communautaire, il est tout aussi impossible de parler de désintérêt, de désaffectation "post-moderne ». Dans le présent article, nous essaierons de voir plus clair en ce qui concerne la vie associative et les solidarités sous-jacentes, à partir d'une recherche sur les associations de loisirs au Saguenay. Après avoir exposé rapidement la méthodologie de notre enquête, nous ferons état des éléments qui vont dans le sens de l'individualisme, au sens strict ou à l'intérieur d'un groupe, pour relever dans un second temps ceux qui contribuent à la formation de réseaux et $d^{\prime}$ une dynamique communautaire, et conclure à leur rencontre et à une prise en charge communautaire des " affaires sociales ».

Bien que sociabilité et solidarité soient des concepts distincts, il est difficile de les aborder séparément; de plus, les deux ont partie liée avec un troisième, celui d'identitét ${ }^{4}$. Solidarité $=$ entraide. Sociabilité $=$ rencontres sociales "désintéressées " selon Simmel (1949). Mais selon cet auteur, on "sociabilise » entre semblables; la sociabilité présuppose une identité commune. Par ailleurs, de qui est-on solidaire ? De ses proches, premièrement, de ceux que l'on fréquente le plus, donc de ceux avec qui on vit la sociabilité. Toutes les études empiriques sur l'entraide montrent que l'échange matériel et l'échange symbolique sont étroitement liés, que sociabilité et solidarité sont les deux faces d'une même médaille. Par ailleurs, on est aussi solidaire de ses semblables, de ceux avec qui on partage une identité commune, des problèmes communs. Dans ce qui suit, nous essaierons dans la mesure du possible de distinguer ces concepts pour mieux éclairer leurs liens. L'objectif de cet article est simplement d'illustrer une dimension méconnue de "l'aide naturelle », l'apport de diverses associations de «loisir ».

\section{Aperçu méthodologique}

Nous avons voulu cerner la sociabilité associative dans la région du Saguenay. Nous avons privilégié la méthode d'entrevue pour avoir accès à la façon dont se vit la sociabilité. En tout, 49 entrevues ont été réalisées (dont 8 doubles, pour un total de 57 informateurs) auprès d'informateurs ${ }^{3}$ âgés de 25 à 79 ans, entre février et août 1990. Nous les avons interrogés sur leurs parenté, milieu de travail, loisirs, participation à des associations formelles et informelles, sur la distribution géographique de leur parenté et de leurs amis, sur les échanges divers qu'ils nouent avec ces différents groupes. 
Le choix des associations s'est fait de deux façons. En ce qui concerne les associations plus formelles, nous nous sommes fiés aux répertoires municipaux publiés par les services de loisirs des villes de Chicoutimi, Jonquière, La Baie et Alma; pour les associations sportives, plutôt informelles, nous avons eu recours à la méthode "boule de neige ", qui consiste à partir d'un informateur clé pour en rencontrer d'autres; dans cette démarche, nous avons cependant cherché à diversifier les associations. Grâce à nos informateurs, nous avons rencontré trois types d'associations, qui se situent globalement dans le monde des loisirs : 1- les spécialisées (17 entrevues, dont une double), dont les activités sont centrées autour d'une activité de loisir précise, par exemple : ligues de quilles, club de golf; 2 - les générales (15 entrevues, dont 5 doubles), où deux ou plusieurs activités de loisirs sont offertes aux membres, par exemple : club récréatif, club de retraités; 3 - et les associations multi-fonctionnelles (16 entrevues dont 2 doubles) ${ }^{6}$ offrant des loisirs à leurs membres tout en étant engagées dans diverses "œuvres", par exemple: Chevaliers de Colomb, club Lions.

\section{a) Individualisme ?}

"Ça devient une passion à un moment donné. " $\left(\mathrm{n}^{\circ} 45\right)^{7}$

Questionnés sur les motifs de leur adhésion à une association, nos interlocuteurs évoquent le loisir, ce sur quoi nous ne nous étendrons pas, ou le "bénévolat ", ce qui retiendra longuement notre attention; mais auparavant, il faut mentionner que dans le cas des « clubs de services » (c.-à-d. associations multi-fonctionnelles recrutant dans la classe supérieure), I'adhésion peut aussi se faire pour des raisons professionnelles.

"L'idée première, c'est que dans un bureau professionnel, il faut à un moment donné que tu connaisses des gens, que des gens te connaissent. Et une des meilleures façons que les gens te connaissent, c'est de faire partie d'un club. [...] C'est une occasion qui est autre, à part le bureau, de rencontrer des gens d'affaires. " (n"33)

«...pour mon travail, premièrement, l'implication du bureau. [...] Le but, notre but, c'est, mon but premier c'est ma job. [...] J'ai rentré 4 personnes de ma famille là-dedans. [...] Moi je suis impliqué à $100 \%$. [...] J'ai toujours aimé le sens de l'organisation. Puis ce que je retire du club; je pense que c'est une œuvre qui est humanitaire. On aide les personnes défavorisées [...], puis je pense que c'est une activité qui vaut vraiment la peine. " ( $\mathrm{n}^{\prime \prime 29)}$ 
Ce dernier exemple est intéressant, car il est tout en ambivalences et illustre bien que différentes logiques et divers motifs d'adhésion à une association, loisirs, bénévolat, sociabilité familiale, relations d'affaires, peuvent se combiner et se renforcer; ils ne sont pas mutuellement exclusifs.

En première approximation, le bénévolat dans l'association est de l'altruisme; cependant, la réalité est complexe et des distinctions s'imposent. Nos informateurs, pour leur part, y incluent aussi bien la participation au conseil d'administration d'un organisme sans but lucratif que l'organisation de soupers-bénéfices ou de tournois sportifs. Pour y voir plus clair nous dissocierons dans ce qui suit deux types de bénévolat. Nous réserverons ce terme pour désigner l'action dans la communauté, par opposition au « travail associatif », effectué dans le cadre de l'association comme organisation. Il faut cependant garder à l'esprit que nos informateurs utilisent le terme bénévolat dans les deux sens, et que dans les deux cas il y a l'idée de don et d'engagement.

Voyons d'abord ce qu'il en est du travail associatif. L'organisation a ses exigences, et quelqu'un qui a du " potentiel » atteint très vite les postes de responsabilité, souvent deux ou trois ans seulement après son adhésion, c'est-à-dire qu'il a tout juste le temps de se familiariser avec l'association, ses membres et ses activités.

" Ils nous détectent, c'est pas long. [...] À la fin de l'année, ils ont dit : toi, t'es capable ! » (n"20a)

Si cela ne surprend guère dans le cas des associations de petite taille où le règlement oblige en plus la rotation des tâches, cela étonne dans les associations de quelques centaines de membres où le bassin de recrutement de responsables est plus important. Cela peut s'analyser comme la rencontre d'une offre et d'une demande. Il y a offre de compétences de la part de ceux qui les ont développées.

"Automatiquement j'avais le goût, avec mon expérience chez les scouts, de travailler dans l'organisation... » ( $\left.\mathrm{n}^{\circ} 17\right)$

"J'ai toujours été un gars qui a aimé travailler dans les organismes. " $\left(n^{\circ} 40\right)$

Dans le cas de gens passant d'une association à l'autre au fil des ans et de leur cycle de vie, il est possible de parler de "carrières associatives ". Vingt-cinq personnes dans notre échantillon sont membres de deux associations ou plus; dix-neuf ont déjà été membres d'autres associations; en tout, ce sont 34 personnes qui n'en sont pas à leur première association ou qui participent simultanément au moins à deux. L'expérience acquise dans un secteur du 
monde associatif peut être mise à profit dans un autre et il n'est pas exceptionnel que des responsables soient recrutés directement, en tant que responsables, dans la mesure où ils sont connus pour détenir cette compétence et cette expérience organisationnelle. En effet, la demande de compétences est grande.

"Ils savaient que j'étais organisateur un peu. Ça fait que mon cousin de Jonquière me demande de partir la loge ici... " ( $\left.n^{\circ} 21 b\right)$

La demande rencontre-t-elle l'offre ? Seulement au prix de sollicitations actives, car le travail associatif est très exigeant. Ces exigences sont de plusieurs ordres. Les membres du conseil d'administration, ou en général les responsables de ces clubs et associations, y consacrent un très grand nombre d'heures par semaine; vingt heures, plus même, ce n'est pas rare. Dans ce cas, le travail associatif est un véritable travail, non rémunéré. Le grand nombre d'heures en jeu explique en partie les difficultés de recrutement pour ces postes et le fait qu'on ait recours à la sollicitation directe; on comprend pourquoi.

"Un membre de l'exécutif peut pas servir deux maîtres. Dans la constitution c'est précisé qu'un membre de l'exécutif ne peut pas faire partie d'un autre exécutif. » $\left(\mathrm{n}^{\circ} 7 \mathrm{a}\right)$

Par ailleurs, la question de la disponibilité est-elle une fausse question ? Ne faut-il pas aussi tenir compte de la motivation ?

"Remarque, je dis que les gens sont affairés, je devrais pas dire ça, parce que la plupart des gens qu'on retrouve dans les clubs Optimistes, c'est tout des gens qui auraient pas le temps. [...] moi, juste pour te donner un exemple, de ce temps-ci, je dois faire en moyenne, par semaine, pour mon travail, autour de 7580 heures par semaine. Ça fait qu'il m'en reste plus ben-ben des heures pour mettre dans l'Optimiste, puis pour mettre ailleurs, mais je trouve le moyen de mettre de l'Optimiste à travers cela. » $\left(n^{\circ} 25\right)$

Et puis, si le travail associatif est du travail non payé, cela suppose une autre source de revenu, d'autant plus qu'il peut même entraîner des coûts pour le "bénévole".

"Quand on fait du bénévolat, $c^{\prime}$ est pas rien que notre temps que l'on donne, c'est aussi notre argent. » ( $\left.\mathrm{n}^{\circ} 20 \mathrm{~b}\right)$

Le travail associatif exige du temps, de l'argent, et quoi encore ? Un bon caractère ! De l'entregent, l'ouverture aux autres.

«Parce que du bénévole, c'est pognant, c'est prenant. T'sé le soir, $t^{\prime}$ as une réunion tel soir. Comme à Québec, faut qu'on y aille 4 fois par année. [...] Pis il y a toujours le chialage; il y en a qui sont pas capables d'accepter l'avis des autres. [...] Des fois, il y en a un qui chicane; woh! il faut que tu l'acceptes. [...] J'aime ça 
m'impliquer. J'aime ça, c'est tout. Je suis fait de même. J'aime ça. » (n'24)

L'association ne profite pas que des compétences personnelles de ses membres; leurs réseaux peuvent — et doivent être mis à profit, nous y reviendrons, mais évoquons pour le moment un exemple : un homme a été recruté en tant qu'intermédiaire entre son beau-frère membre de l'exécutif et son frère disposant d'un camion dont l'association avait besoin ( $\left.\mathrm{n}^{\circ} 25\right)$.

Une fois établie la liste des exigences du travail associatif, on en arrive à se demander ce qui pousse à s'y engager. C'est qu'il apporte néanmoins des gratifications personnelles. Selon Dumazedier (1990), le fait que ces gratifications soient explicites, et que la personne ne travaille pas uniquement en vue d'une cause collective, même si celle-ci demeure importante, serait nouveau; il parle même de néo-bénévolat. Quelles sont donc ces gratifications? Certains en retirent un enrichissement, un changement personnel, qu'ils évoquent sans spécifier.

"Aux personnes âgées, on donne pas d'argent, on donne du temps, puis on aime ça. On a ben du plaisir. On aime beaucoup ça. C'est sûr qu'il y a des gens qui sont très « social ", qui le seront toujours [...] mais pour la plupart je dirais, pour la plupart qui font ça, ça leur ouvre effectivement, ça change un petit peu leur vie. [...] Tu deviens... tu deviens.. ça change. C'est mon cas personnellement. " ( $\left.\mathrm{n}^{\circ} 33\right)$

C'est l'occasion également de faire des apprentissages qu'ils n'auraient pas eu l'occasion de faire ailleurs. Ces apprentissages recoupent en partie les changements personnels dont nous venons de parler, mais par opposition à ces derniers, ils sont plus formels : acquisition de connaissances et $d^{\prime}$ expérience en matière d'organisation ou d'administration. Pour poursuivre la métaphore économique, il y a non seulement offre et demande de compétences, mais formation de la main-d'œuvre.

"On diversifie nos activités en masse. [...] Puis ceux-là qui sont intéressés à s'impliquer, ils [...] vont apprendre des choses. Moi j'ai appris beaucoup. » ( $\left.n^{\circ} 39\right)$

"Partager de l'expérience, mais surtout aussi pour prendre de l'expérience. » ( $\left.\mathrm{n}^{\circ} 48\right)$

Le travail associatif dans une association est un don à l'association qui implique en retour un contre-don (Mauss, 1950), une obligation envers ceux qui l'effectuent.

" Actuellement, on est 72 campeurs. On a 50-55 membres puis 2530 invités. Il y a des invités qu'on a gardés, qu'on n'a pas voulu 
mettre dehors, parce qu'ils nous ont aidés; ils ont contribué à la construction de ce qu'on est actuellement. " ( $\left.n^{\circ} 43\right)$

L'association peut s'acquitter de différentes façons de sa dette envers ceux, membres ou non-membres, qui lui fournissent de l'énergie; elle peut ainsi organiser un souper, une fête pour les bénévoles. Enfin ce travail associatif, d'organisation des loisirs, devient une sorte de loisir.

"Chacun prend ses responsabilités bien à cœur. [...] Il y a beaucoup de plaisir à travailler ensemble. » $\left(n^{\circ} 48\right)$

À tel point que la corvée peut sembler une partie de plaisir !

"Par exemple, l'an passé, ils ont dit, le Conseil, il faudrait rafraîchir la bâtisse. [...] On a peinturé ça, on a refait les planchers. Ça a été rien que du bénévolat (pendant un mois et demi), ça a été rien que du plaisir. Mon beau-frère de Hull était ici, il est venu deux jours avec moi. Ce gars-là, il rit pas chez lui, ici [Untel] a réussi à le faire rire. " $\left(n^{\circ} 4 a\right)$

Sans se muer nécessairement en partie de plaisir, c'est un travail qui a un sens, et même plusieurs sens.

"C'était pour mieux se connaître, pour mieux fraterniser dans le travail, et comme social aussi... [...] Alors je venais ici pour travailler, rencontrer les gens. J'aime beaucoup rencontrer les gens : on discute, on parle. C'était ça surtout, la rencontre, pas la compétition. " ( $\left.\mathrm{n}^{\circ} 12\right)$

Il est important de remarquer que travail et loisir ne s'opposent pas. Le loisir, collectif, apporte bien sûr du plaisir à chacun; quant au travail associatif, il comporte de lourdes exigences et responsabilités, mais fournit à ceux qui l'effectuent des gratifications personnelles, qui vont du plaisir à la formation en passant par l'obtention de droits. À ce titre, on pourrait y diagnostiquer de l'individualisme. Cependant, ces gratifications individuelles ne vont pas à l'encontre des objectifs de l'association et de son fonctionnement collectif; peut-on alors parler d'individualisme de groupe ? La prochaine section explorera cette rencontre des membres et de leurs objectifs personnels dans l'association.

\section{b) Individualisme de groupe ?}

Pour que la vie associative soit un succès, tant dans sa dimension organisationnelle que dans la sociabilité, il doit y avoir intégration des membres. L'intégration au groupe est à la fois cause et conséquence de l'adhésion, cause et conséquence de la vie associative. Voici un exemple où l'intégration au groupe est un but recherché au moment de l'adhésion. 
" Je connaissais pas de monde ici à Jonquière [...] C'est pour ça que je me suis impliqué : faire des contacts, faire du social. » $\left(n^{\circ} 11\right)$

Les associations informelles ont des critères d'adhésion implicites, relevant de la sociabilité; l'adhésion alors est conséquence de l'intégration préalable à un groupe. Voici l'exemple d'une association de dégustateurs de vin :

"Les gens sont un peu choisis au départ, on les connaît, c'est des bons amateurs, mais qui ne font pas d'esclandre; il n'y a jamais eu de problème [d'ivresse]. [...] Aux soupers, on dit " si vous avez parmi vos amis des gens qui seraient intéressés ". Plusieurs personnes font partie d'un club social ou l'autre, Kiwanis, Richelieu, etc., donc pour eux c'est une autre activité, peut-être pas conséquente l'une à l'autre, mais c'est des gens qui connaissent du monde. " $\left(\mathrm{n}^{\circ} 5\right)$

L'intégration, même si elle préexiste à l'adhésion, est nécessairement renforcée par la participation intensive ${ }^{8}$. Son envers, $c^{\prime}$ est qu'il peut être difficile de mettre le pied dans l'engrenage.

"C'est sûr que nous autres on est pas hermétiques dans le sens que ça fait longtemps qu'on est ici... on se pose plus les questions d'intégration, $t^{\prime}$ sé. Mais les gens qui arrivent, ils trouvent ça difficile les premiers temps. " $\left(n^{\circ} 41\right)$

En effet, n'importe quel groupe ne fait pas l'affaire de n'importe qui, cela prend un minimum d'affinités, ce qui nous renvoie à l'intégration minimale qui doit être présente dès le départ, pour adhérer. La participation aux activités du groupe permet de développer ce sentiment d'appartenance; c'est véritablement une rétroaction positive.

"C'est des belles places pour du monde comme nous autres.

D'abord, la plupart des gens, c'est des gens comme nous autres, c'est du monde qui aime se divertir autrement dit, pis se rencontrer. Moi j'aime ben ça. Ma femme est ben satisfaite de ça aussi. » $\left(n^{\circ} 13\right)$

L'intégration profite au groupe, en lui fournissant la cohésion dont il a besoin pour le succès de ses activités, et au membre pour qui l'intégration peut paradoxalement être l'occasion d'une ouverture à un milieu plus vaste :

"Je suis bien content d'ailleurs d'être rentré dans le club Lions. Moi personnellement, ça m'a apporté, dans le sens, beaucoup d'ouvertures; je suis un type assez gêné, à peu près incapable de prendre la parole devant le monde. Je fonctionnais, je faisais mes affaires, sauf que ça m'a fait une ouverture, ça m'a donné beaucoup d'ouverture sur les gens, sur la société, beaucoup de confiance en moi aussi. " ( $\left.n^{\circ} 23\right)$ 
Le groupe rassemble, et du coup permet la cristallisation des liens individuels qu'il suscite et renforce en une identité commune. L'adhésion à une association peut servir de tremplin pour une intégration non seulement au groupe en question, mais à la communauté, en particulier à une nouvelle communauté à la suite d'un déménagement ou grâce à l'acquisition de nouvelles compétences. Mais si on n'est pas déjà minimalement intégré au groupe, si on n'y a pas au moins une ou des "connaissances ", il est difficile de s'y sentir à l'aise. C'est une "structure structurante $»^{9}$; il s'y joue une rétroaction positive entre l'adhésion et l'intégration. L'association est un espace de sociabilité ${ }^{10}$ privé-collectif, lieu à la fois de consolidation d'identités déjà existantes, en particulier familiales étant donné le recrutement qui se fait sur la base des réseaux de sociabilité, et de formation de nouvelles identités.

L'intégration est une fermeture - créatrice ? - du groupe sur lui-même, mais pas synonyme de retrait de l'individu de la communauté; de façon analogue, on peut se demander si l'intégration du groupe favorise le repli du groupe sur lui-même ou une ouverture sur la communauté.

\section{c) Le réseau associatif}

Existe-t-il une communauté à laquelle s'identifient ou du moins se rattachent les diverses associations rencontrées, ou chacune évolue-t-elle en vase clos, dans une bulle de sociabilité ? Peut-on parler de réseau associatif ? Pour le faire, il faudrait qu'existe un lien entre des partenaires, et qu'à la faveur de ces liens quelque chose circule.

Le shuffleboard c'est pareil. C'en est une de notre ligue qui a parti la ligue aux Moose. [...] Ça a commencé à Aramis, ça a été à I'ARCN, ça a été aux Moose, puis après ça le Patro et Saint-Bruno. $\left(n^{\circ} 4\right)$

Les liens susceptibles d'unir les associations sont divers. Ce peut être d'abord une activité autour de laquelle on organise des tournois ou des manifestations en collaboration.

On a des liens, quasiment, avec toutes les salles. Comme nous autres, on joue aux Chevaliers de Colomb. On a un lien, ni plus ni moins. On a un lien avec la Légion canadienne, vu qu'il y a une ligue de dards là. On a des liens avec les Moose, vu qu'il y a une ligue de dards aux Moose. $\left(n^{\circ} 24\right)$

Les associations doivent se concerter en ce qui concerne leurs horaires, pour ne pas toutes organiser leurs repas-bénéfices en même temps par exemple; c'est un ajustement technique, mais le lien sous-jacent $n^{\prime}$ est-il que technique? 
"On s'organise tout le temps pour pas que notre souper arrive à la même date que l'autre souper de l'autre salle. " ( $\left.{ }^{\circ} 24\right)$

"On est en contact assez régulièrement avec la Légion canadienne, les Chevaliers de Colomb aussi. [...] On s'entraide. [...] Même quand c'est le temps d'organiser des activités, on s'en parle, pour pas rentrer en conflit. C'est parce qu'on a des membres qui font partie des deux. " ( $\left.n^{\circ} 11\right)$

La concertation relative aux horaires peut aller plus loin que la planification d'une activité ponctuelle, par exemple de retarder la saison de dards à cause du grand nombre de chasseurs membres de ligues de dards.

Les associations peuvent collaborer à l'organisation non seulement d'activités de loisirs, mais aussi de bonnes œuvres, de campagnes de financement. Ces catégories ne sont pas exclusives; l'organisation d'une activité commune peut prendre couleur de bonne œuvre, comme le révèle l'exemple d'une association qui organise un tournoi de billard contre une autre qui s'occupe $d^{\prime}$ ex-alcooliques $\left(n^{\circ} 21\right)$. Des associations peuvent aussi s'échanger des services; ainsi les clubs de l'Âge d'or peuvent avoir accès gratuitement à la salle des Chevaliers de Colomb, ou un club de photo consentir des rabais aux autres organismes sans but lucratif.

« Depuis 2 ans, l'aspect social est beau [...] On rencontre beaucoup de gens, on parle beaucoup de photo. [....] Ça nous a permis de connaître beaucoup de gens d'autres associations, surtout les gens des C.A. [...] On les rencontrait, c'était Monsieur Tout le Monde. Maintenant $c^{\prime}$ est Madame de I'AFEAS, Madame de l'Académie de ballet...» $\left(n^{\circ} 32\right)$

Le savoir-faire que les groupes se transmettent, comme dans le cas du shuffleboard évoqué plus haut, est un cas particulier de service.

À ce stade, nous ne pouvons que constater l'existence de liens entre les associations, mais il n'est pas encore possible de parler de réseau associatif. Notons toutefois que ces liens sont de deux genres : les liens obligés, ceux que la concertation impose, et les échanges de services entre groupes ou l'organisation d'activités en collaboration, qui sont facultatifs. La concertation est nécessaire non seulement parce que le public convié à diverses activités est le même, mais parfois parce que les membres sont les mêmes.

Quelques remarques en ce qui concerne les partenaires échangistes. Le savoir-faire se transmet entre associations aux activités analogues, tout comme les tournois s'organisent entre associations qui ont en commun une activité; mais la concertation des horaires doit se négocier entre associations susceptibles de recruter la même clientèle de membres ou d'invités; cela mobilise un 
réseau plus large, tout comme l'échange de services. Une activité commune entre associations (tournois, partie de sucre, par exemple) se tient à la faveur des liens existant entre les responsables des associations, mais met en présence les membres de la base, ce qui peut relancer d'autres idées de collaboration.

Après avoir parlé du lien et des partenaires, parlons de ce qui crée le lien; nous avons vu plus haut qu'il peut s'agir d'une activité autour de laquelle on organise rencontres et tournois. Ce peut être aussi une personne qui est membre de deux associations, par exemple un membre du C.A. d'une association qui est aussi membre " ordinaire " d'une autre.

"On joue aux dards aux Chevaliers de Colomb, mais [je ne suis] pas Chevalier. Ils ont toujours voulu me rentrer, mais j'ai jamais voulu, j'en avais assez. " $\left(n^{\circ} 13\right)$

Une autre possibilité, c'est que les membres d'un réseau de parenté participent à plusieurs associations. Le lien sera donc informel, mais non moins réel. Des informateurs ont ainsi un frère Élan ou Chevalier de Colomb, sans eux-mêmes appartenir à cette association. La distance peut aussi faire qu'un homme soit membre d'une loge de Moose à Jonquière et son père à Alma, ou Chevalier de Colomb à La Baie et son frère à Jonquière, etc.

Il faut insister sur le rôle structurant de l'espace physique dans la création de l'espace de sociabilité ${ }^{11}$, cela ne concerne pas que "l'interne", mais aussi les relations entre les associations.

"Prends, comme anciennement, quand on était au sous-sol de l'église Ste-Marie, nous autres, les préretraités, il y avait l'âge d'or de Ste-Marie, pis après ça, il y avait plusieurs associations : les femmes de l'AFEAS, c'est toutes des affaires de même. [...] Des fois on organise un voyage [...] s'il manque des gens, on peut appeler aux préretraités. Faut s'aider. » ( $\left.n^{\circ} 13\right)$

$S^{\prime}$ il semble possible d'évoquer un réseau associatif, la question se pose : ce réseau agit-il en circuit fermé ou s'ouvre-t-il sur une communauté plus large que celle de ses membres? Ce qu'il est possible d'affirmer pour le moment, c'est que le réseau associatif existe; il se constitue à la faveur tant de la logique associative que des réseaux de sociabilité des membres, les deux se recoupant et se renforçant mutuellement. La section suivante est entièrement consacrée à une des activités qui tissent les liens à la fois entre les associations et entre celles-ci et l'ensemble de la communauté.

\section{d) Du réseau à la communauté}

"Le club Optimiste de la Baie, par exemple, a fourni une personne pour le C.A., un autre va aider financièrement un groupe, 
le groupe de sa ville. Les Chevaliers de Colomb montrent une très grande ouverture. On leur rend service, on vend leurs lapins de chocolat. " $\left(n^{\circ} 45\right)$

Indépendamment des liens entre associations, une chose qu'il faut analyser soigneusement pour statuer sur l'existence - ou non - d'une dynamique communautaire ce sont les « œuvres" dont s'occupent les diverses associations. Les « œuvres » sont davantage le fait d'associations multi-fonctionnelles, mais pas nécessairement; ainsi un club canin visite les personnes âgées, une chorale présente des récitals dans les hôpitaux et centres d'accueil. Par définition, ces « œuvres » constituent l'ouverture des associations sur la communauté. Voyons comment cela s'effectue.

Nous parlerons ici de toutes les formes d'aide que les associations apportent : institutionnelle ou informelle, dirigée vers les membres ou les non-membres, sous forme d'argent ou d'activités. II faut donc distinguer plusieurs cas. Premièrement, I'aide aux membres de la part de l'association, informelle et financière.

«On vient en aide à nos membres aussi qui sont dans la dèche temporairement. C'est confidentiel. La demande me parvient à moi. Je m'occupe strictement de ça sous serment pour que personne sache qui on dépanne. " $\left(n^{\circ} 9\right)$

II n'est pas toujours facile de démêler cela de l'entraide entre membres, informelle et qui peut prendre la forme de services ou de prêt d'argent :

" Une fois j'ai rencontré quelqu'un icitte qui avait besoin d'un chauffeur. Il a donné mon nom et j'ai rentré pour la compagnie. Pas longtemps, mais j'ai rentré. C'est lui qui m'a fait rentrer. On $\mathrm{s}^{\prime}$ aide beaucoup entre nous quand on peut. " ( $\left.\mathrm{n}^{\circ} 10\right)$

Les " clubs de services» ne sont pas des associations de gens d'affaires, mais presque; I'entraide y est en quelque sorte statutaire.

"Si moi j'ai un garage de vente d'automobiles, je vais m'attendre à ce que les membres du club viennent. " ( $\left.n^{\circ} 26\right)$

Cette entraide entre membres d'un " club de services " peut même être une des causes d'adhésion. Cela dit, l'entraide informelle entre membres est susceptible d'exister partout où il y a de la sociabilité, et une association solidaire aidera ses membres en difficulté.

Mais les associations ne se limitent pas à aider leurs membres. La " charité » envers les non-membres est plus facile à caractériser; elle peut prendre la forme de "secours direct » plutôt informel, comme dans les exemples suivants, ou de campagnes de souscription, formelles. En effet, pratiquement toutes les associations 
multi-fonctionnelles ont une cause prioritaire, que ce soit l'aide à la jeunesse en général ou à la jeunesse inadaptée, l'aide aux personnes atteintes d'une maladie en particulier, comme la fibrose kystique. Elles peuvent aussi avoir leur fondation, comme les Élans, ou venir en aide à celle d'un hôpital ou d'une cause comme MIRA. Les associations peuvent encore s'impliquer dans l'organisation d'un téléthon. Bref, la charité peut être institutionnelle ou informelle, ponctuelle.

«Récemment encore, on a acheté 3-4 paires de lunettes pour des jeunes qui avaient besoin de lunettes, pis que les parents étaient pas capables de payer. On est beaucoup référé par les hôpitaux, les optométristes, pis ces choses-là, pis quelquefois aussi par des gens qui connaissent l'association, qui eux nous appellent directement. » $\left(n^{\circ} 18\right)$

Ce type d'aide informelle est effectué grâce à des budgets généraux de fonctionnement. Quand les "œuvres "sont institutionnelles, l'association organise des collectes de fonds, des campagnes de souscription. Plusieurs modalités de financement sont possibles. Certaines associations, pour rejoindre l'ensemble de la communauté, misent sur le porte-à-porte.

«Des levées de fonds, des campagnes, avant ça, on en faisait 1012; on les faisait toutes. Maintenant, on s'est limités à 3 ou 4. [...] Il était une secousse, on se promenait en ville, ils nous appelaient les quêteux. " ( $\left.\mathrm{n}^{\circ} 21 \mathrm{~b}\right)$

Mais le plus souvent, pour recueillir des fonds, I'association organise une soirée ou un repas-bénéfice. Deux remarques : tout d'abord les donateurs reçoivent quelque chose en échange de leur contribution financière, on est dans une logique de don (Mauss, 1950), et deuxièmement les billets sont vendus selon les filières des réseaux de sociabilité des membres. Le repas-bénéfice est une façon de combiner l'utile et l'agréable; les membres qui vendent des billets pour ces occasions misent sur la sociabilité : ils y invitent parents et amis, et en même temps ils misent sur la solidarité communautaire de ceux-ci à l'égard de la cause ainsi financée. Lorsque ce que l'on finance ainsi ce sont les activités courantes d'une association, par exemple avec le brunch-bénéfice d'une chorale, ce qui s'exprime c'est avant tout la sociabilité, mais le plus souvent le repas-bénéfice sert à recueillir des fonds pour une cause plus large que celle du fonctionnement du groupe. En règle générale la solidarité à l'œuvre à l'occasion de ces repas-bénéfices est autant interpersonnelle que communautaire :

"Regarde, C'est un confrère, $X$, qui est avocat, qui est dans le club Richelieu; alors lui me sollicite pour acheter deux billets 
à $35 \$$. Moi j'ai dit " $M^{\prime}$ a t'en prendre; tu vas $\mathrm{m}^{\prime}$ en acheter deux à $25 \$$ ». $C^{\prime}$ est du prenant-prenant. » ( $\left.n^{\circ} 16\right)$

$\mathrm{D}^{\prime}$ autres associations, par la diversité professionnelle de leurs membres, rejoindront l'ensemble des catégories professionnelles représentées au sein de l'association. Cette stratégie semble a priori viser moins un réseau de sociabilité qu'un réseau professionnel. Mais le réseau des professionnels est avant tout un réseau de professionnels, moins lié à la parenté qu'au couple et aux relations du mari (Fortin et al., 1987). En ce sens, c'est toujours sur les réseaux de sociabilité des membres que repose le succès des collectes de fonds, à l'exception de celles qui s'effectuent par le porteà-porte. Quelle que soit la dynamique de vente de billets, le groupe s'affirme en tant que groupe solidaire, à l'interne, car cela révèle au grand jour la sociabilité de ses membres et, à l'externe, par rapport à la communauté et à la cause défendue.

L'aide apportée par les associations n'est pas que matérielle et financière, mais aussi psychologique, c'est un réconfort moral. Ce qui est alors donné, c'est du temps, comme lorsqu'une chorale présente un concert dans un centre d'accueil ou un hôpital, et sont beaucoup moins impliqués (mais rarement pas du tout) les réseaux des membres que dans le cas de collectes de fonds, ce qui contribue à l'invisibilité sociale de ces pratiques.

Les « œuvres" auxquelles les associations étudiées consacrent leurs énergies sont une forme de prise en charge des problèmes de la communauté par la communauté. Il s'agit surtout d'aide financière, mais aussi de services. De plus, par la mobilisation de leurs réseaux, les associations sensibilisent une fraction non négligeable de la population à ces problèmes.

La communauté qui prend charge se situe en dehors du réseau des affaires sociales (celui qui fait du « développement communautaire "), et même des groupes populaires et communautaires ${ }^{12}$. Cette communauté a conscience d'exister et se donne des occasions de manifester, de "célébrer»son existence.

"II y a tout un but commun [avec d'autres associations], c'est de servir la communauté. On est tous là pour aider; il n'y a pas de club qui est là juste pour le social; il est là pour faire des campagnes de financement, pour aider les jeunes. Le Club Optimiste est là pour aider les jeunes; le Club Rotary, eux autres aident, je me souviens plus quelle catégorie d'âge, mais on a certaines affinités. À tel point que l'an passé, on a fait un souper réunissant tous les clubs sociaux de la région de Chicoutimi-Jonquière. Alors ça a réuni 300, 400 ou 500 personnes; on a pu échanger, partager. » ( $\left.\mathrm{n}^{\circ} 16\right)$ 
Les associations de loisir mettent en œuvre une charité publique invisible, ou presque, mais importante, secourant leurs membres aussi bien que les non-membres. Pourquoi "invisible»? Parce que ce n'est pas à cela qu'on pense quand on évoque ces groupes, à tel point que les réflexions sur le "communautaire » et les solidarités menées par les sociologues ou les travailleurs sociaux les oublient le plus souvent.

\section{Conclusion : solidarités et prise en charge communautaire}

Dans la vie associative se rencontrent des logiques individuelle, de groupe et communautaire qui se renforcent mutuellement. Le réseau de l'association, c'est-à-dire les relations que le groupe en tant que tel peut mobiliser, ne se confond pas avec ceux de ses membres, mais les recoupe largement.

L'association ne se substitue pas aux réseaux individuels des membres, car elle mise sur ces réseaux pour le succès de ses activités, surtout celles de financement, mais aussi de recrutement. Cette analyse nuance les propos de certains auteurs (par exemple, Levasseur, 1990), selon lesquels la vie associative tendrait à se substituer aux sociabilités traditionnelles. Quelles sont, précisément, les solidarités mises en œuvre?

Quand il s'agit de l'entraide entre membres, les frontières du groupe d'appartenance coïncident avec celles de l'association. Mais plusieurs non-membres sont aidés (matériellement et psychologiquement) et l'aide vise l'ensemble de la communauté. Par le truchement de la sollicitation de leurs réseaux (vente de billets) ou de l'ensemble de la collectivité (porte-à-porte), les membres des associations entraînent d'autres qu'eux-mêmes dans cette charité publique; il serait sans doute exagéré de dire qu'ils mobilisent l'ensemble de la communauté pour se prendre en charge, mais la mobilisation effectuée va bien au-delà des frontières de l'association. De plus, ils ne se contentent pas de sensibiliser, mais recrutent des bénévoles, collectent des fonds. Dans le cas où l'association donne de son temps, des services à la communauté, c'est elle-même qui se mobilise et qui affirme ainsi son appartenance à un groupe plus large. Nous avons parlé plus haut de la cohésion du groupe, de l'intégration des membres; cela n'est pas contradictoire avec l'ouverture du groupe sur la communauté. C'est dans la mesure où ils sont bien intégrés au groupe qu'ils seront mobilisés par la cause privilégiée par celui-ci. L'appartenance à une association débouche souvent sur l'appartenance à une communauté. 
Poser la question de l'appartenance à une association amène à s'interroger sur le mode de recrutement des associations. Recrutent-elles en fonction de l'appartenance à un réseau (identité globale) ou d'une activité (identité partielle) ? Qu'une association regroupe une clientèle bien ciblée ne signifie pas nécessairement qu'elle la recrute en fonction d'une identité partielle. Des associations au membership très ciblé - comme des retraités - peuvent recruter leurs membres comme personnes entières, c'est-à-dire non simplement en tant que retraités de telle ou telle compagnie, mais en tant que personnes intégrées dans des réseaux, dont les membres sont appelés à devenir "membres-auxiliaires » ou bénévoles... Des associations de personnes âgées peuvent ainsi être à la fois des lieux où on joue aux cartes et des groupes de pression. De façon analogue, que l'activité soit spécialisée ne veut pas dire qu'on y adhère selon une identité partielle. Prenons l'exemple des quilles : on y joue essentiellement avec les membres de son réseau, celui-ci s'enrichissant de nouveaux membres connus aux quilles. C'est une activité très spécialisée, mais qui recrute des membres d'un réseau avant que des amateurs. Ce n'est pas le cas cependant de toutes les associations spécialisées; ainsi on joue au scrabble entre scrabbleurs.

Que l'association soit ciblée ou non, spécialisée ou non, souvent $y$ adhère, en une seule personne, le père ou le frère qui recrutera son fils ou son frère, le bénévole qui collaborera aux activités, le travailleur qui vendra des billets pour un souper-bénéfice à ces confrères, le conjoint qui mobilisera sa femme pour l'organisation de ce souper, etc. Le groupe n'est pas fermé sur luimême. Toute la personne est mobilisée et non seulement une de ses identités, par opposition par exemple aux Alcooliques anonymes, cas typique de groupe d'entraide. Est-ce le cas de toutes les associations que nous avons rencontrées ? Non, mais nous pouvons affirmer que dans l'ensemble les associations de loisir ne remplacent pas les réseaux "traditionnels »- basés essentiellement sur la parenté et dans une moindre mesure sur le monde du travail - mais "sont assises dessus »: elles misent sur eux pour leur constitution.

La taille des agglomérations saguenayennes, où " tout le monde se connaît », peut renforcer l'impression que la vie associative y est presque "naturelle » et déborde non moins "naturellement » sur la vie communautaire. En l'absence de données comparables pour d'autres régions du Québec, il est impossible de dire s'il s'agit d'une spécificité régionale ou non. Toutefois, la taille de la région et sa situation périphérique produisent dans d'autres 
domaines des effets spécifiques (ainsi dans le milieu de l'art: Tremblay, 1981). Bouchard, dans une synthèse des travaux sur le Saguenay (1986), caractérise ainsi la dynamique communautaire en milieu rural au $19^{\mathrm{e}}$ siècle et au début du $20^{\mathrm{e}}$ : 1 - les solidarités n'y sont pas une dysfonction, mais un effet de la marginalité; $2-$ on vit en symbiose avec l'économie dominante; 3 - on répond à des impératifs sociaux plus qu'à la recherche de profits; 4- les solidarités sont non seulement micro-locales, mais aussi nationales et internationales; 5 - donc les solidarités ne sont pas synonymes, au contraire, d'enracinement; 6- ce n'est pas tant une culture du refus du présent qu'une culture de la solidarité. La vie associative que nous avons observée se situe dans le prolongement de cette culture de la solidarité. Nous pourrions tenter de caractériser les solidarités que nous avons observées d'une façon qui serait aussi en quelque sorte une reformulation-généralisation des caractéristiques de la culture de la solidarité de Bouchard (1986), mettant en évidence sa persistance dans la société actuelle : les solidarités familiales y sont très présentes comme pratiques mais aussi comme représentation; les autres solidarités y sont décrites et vécues sous le mode familial $\left.\right|^{13}$. Ces solidarités sont un lieu à la fois de fermeture du groupe sur lui-même et d'ouverture sur une communauté plus large. Le rapport à l'économie est ambigu : le travail y est important, mais dans une logique sociale plus que financière. Au-delà du cas saguenayen, retenons le passage d'une dynamique de sociabilité privée à des solidarités communautaires. II faudrait étudier plus avant, dans des communautés plus importantes, les possibles et les limites de ce passage et de la solidarité ainsi engendrée.

Revenons en terminant sur la dialectique ouverture-fermeture du groupe sur lui-même. Un membre bien intégré dans le groupe y fera des apprentissages (personnels ou plus formels) qui lui permettront de s'épanouir, de s'ouvrir sur la communauté, dans son milieu de travail. Par ailleurs, l'association a besoin des réseaux de ses membres; pour son recrutement, ce qui est le cas de toutes les associations, tant spécialisées que générales ou multi-fonctionnelles, car semblent constituer l'exception ceux qui y adhèrent sans être sollicités personnellement. L'association qui entreprend une collecte de fonds, pour son propre financement ou pour une œuvre, misera aussi sur les réseaux personnels de ses membres, même chose si elle entreprend une corvée ou a besoin de bénévoles pour une activité ponctuelle. À la limite, on pourrait dire que les associations de loisir recrutent dans les réseaux de leurs membres, et que ce qu'elles recrutent, ce sont les réseaux de leurs nouveaux membres. 
Tous ne sont pas membres d'associations, mais le nombre d'associations va en s'accroissant. La vie associative, même dans le cas d'associations appartenant au monde du loisir et misant sur le plaisir d'être ensemble, met en œuvre des solidarités communautaires, permettant une prise en charge de la communauté par elle-même.

\section{Notes}

1. Cette recherche, effectuée dans le cadre de SOREP, Centre interuniversitaire de recherches sur les populations, et grâce au financement du Conseil de la recherche en sciences humaines du Canada, a donné lieu à un rapport de recherches : Andrée Fortin et David Rompré, Vie associative, solidarités et dynamique communautaire : SOREP, Université du Québec à Chicoutimi et Département de sociologie, Université Laval, Québec, octobre 1991.

2. En 1973, ces trois catégories regroupaient environ les deux tiers des associations actives; les sportives ont depuis diminué au profit des "liens sociaux".

3. Pour une discussion des différences entre ces trois types de groupes, " de loisir ", " politiques" et "d'entraide », voir Fortin, 1991.

4. Ce que nous ne développerons pas dans ce texte, mais qui est central dans notre rapport de recherche, où nous nous interrogeons sur l'identité individuelle et l'identité collective, et leurs transformations dans le contexte du passage à la modernité puis à la post-modernité.

5. Il s'agissait d'informateurs et non d'informatrices à trois exceptions près; cette recherche, en effet, se situe dans le prolongement d'une précédente (Fortin et al., 1987) où nous avions surtout rencontré des informatrices, ce qui avait soulevé des questions sur la spécificité de la sociabilité masculine. Dans cet article, nous n'entrerons pas dans ces considérations.

6. Un informateur n'était officiellement membre d'aucune association, mais bénévole dans celle de son épouse. Les entrevues doubles n'ont pas été planifiées comme telles; c'est lorsque nous nous présentions au rendez-vous, souvent au local de l'association, qu'un second informateur se joignait à nous.

7. Les numéros indiqués après les citations renvoient aux numéros donnés aux entrevues.

8. Rares sont les cas comme les Vétérans où le groupe, non seulement préexiste à l'association (ce serait le cas des clubs de loisirs liés à des entreprises), mais où on se sent des obligations envers les non-membres de l'association, membres du groupe : " S'il a pas sa carte de membre, s'il paye pas sa cotisation [il n'est pas membre]. Mais il arrive une chose. $S^{\prime}$ il décède et on sait que $c^{\prime}$ est un vétéran, on lui fait des honneurs pareil, comme $s^{\prime}$ il était membre. » $\left(n^{\circ} 31\right)$ 
9. À ce titre, on pourrait parler d'habitus associatif; on observe en effet une socialisation familiale à la vie associative; une socialisation de la famille par la vie associative; enfin, cela s'exprime souvent par la participation, simultanée ou successive, à plusieurs associations.

10. Et souvent, en raison de l'existence d'un local, un espace physique privé-collectif.

11. Ce qui n'est pas propre à la vie associative.

12. Klein et Gagnon, 1989, ont étudié les liens entre le " mouvement populaire », en particulier dans sa composante écologiste, et l'État, au Saguenay; voir aussi Anadon et al., 1990, sur le mouvement féministe, toujours dans la même région.

13. Ce que nous n'avons pas eu l'occasion de développer dans cet article; voir Fortin et Rompré, 1991.

\section{Références bibliographiques}

ANAdon, Marta, Dominique MASSON, Marielle Tremblay et Pierre-André TREMBLAY (1990). "Les collectives de femmes : une démocratie sororale ", Nouvelles pratiques sociales, vol. 3, n' 2 : 57-90.

BOUCHARD, Gérard (1986). "La dynamique communautaire et l'évolution des sociétés rurales québécoises aux $19^{\mathrm{e}}$ et $20^{\mathrm{e}}$ siècles. Construction d'un modèle ", Revue d'histoire de l'Amérique française, vol. 40, n 1 : 51-71.

BRAULT, Marie-Marthe T. et Lise SAINT-JEAN, dir. (1990). Entraide et associations. Québec : IQRC, coll. Questions de culture, $\mathrm{n}^{\circ} 16$.

DUMAZEDIER, Joffre (1990). "Pour un renouveau de la recherche en sciences sociales du loisir ", Loisir et société, vol. 13, $\mathrm{n}^{\circ} 1:$ 63-76.

FORTIN, Andrée, avec la collaboration de Denys DELAGE, Jean-Didier DUFOUR et Lynda FORTIN (1987). Histoires de familles et de réseaux, La sociabilité d'hier à demain. Montréal : Saint-Martin.

FORTIN, Andrée (1991). "La participation : des comités de citoyens au mouvement communautaire ", dans Jacques T. GODBOUT, dir., La participation politique. Québec, IQRC, coll. Questions de culture, $\mathrm{n}^{\circ} 17$ : 219-250.

FORTIN, Andrée et David ROMPRÉ (1991). Vie associative, solidarités et dynamique communautaire. Chicoutimi : UQAC, SOREP et Québec : Département de sociologie, Université Laval.

GODBOUT, Jacques T. (1990). "Le retour du social », dans Roger LEVASSEUR, dir., De la sociabilité. Spécificité et mutations. Montréal : Boréal, p. $210-230$.

GRANOVETTER, Mark (1983). "The Strength of Weak Ties : A Network Theory Revisited", dans Randall COLLINS (ed.), Sociological Theory. San Francisco : Jossey-Bass, p. 201-233.

HAMEL, Pierre (1990). "Les mouvements sociaux et le retour de l'individualisme », dans Roger LEVASSEUR, dir., De la sociabilité. Spécificités et mutations. Montréal : Boréal, p. 249-268. 
KLEIN, Juan-Luis et Christiane GAGNON (1989). Le social apprivoisé. Hull : Asticou.

LANGLOIS, Simon, avec la collaboration de Jean-Paul BAILLARGEON, Gary CALDWELL, Guy FRÉCHET, Madeleine GAUTHIER et Jean-Pierre SIMARD (1990). La société québécoise en tendances, 1960-1990. Québec : IQRC.

LEVASSEUR, Roger et Raymond BOULANGER (1990). "La dynamique des associations au Québec : démographie et morphologie : 1942-1981", dans Marie-Marthe T. BRAULT, Lise SAINT-JEAN, dir., Entraide et associations. Québec : IQRC, coll. Questions de culture, n 16 : 153-179.

LEVASSEUR, Roger, dir. (1990). De la sociabilité. Spécificité et mutations. Montréal : Boréal.

MAUSS, Marcel (1950). "Essai sur le don", dans Sociologie et anthropologie. Paris : PUF, p. 145-279.

SIMMEL, Georg (1949). "The sociology of sociability ", American Journal of Sociology, vol. 55 : 254-261.

TREMBLAY, Denys (1981). "Un art régional : avant tout un art d'attitudes", dans Richard MARTEL, dir., Actes du Colloque Art/Société. Québec : Éditions Intervention, p. 98-115. 